\title{
Ginseng as a Treatment for Fatigue: A Systematic Review
}

\author{
Noël M. Arring, DNP, RN, OCN, ${ }^{1, *}$ Denise Millstine, $M D_{1}^{2,3}$ \\ Lisa A. Marks, MLS, AHIP, and Lillian M. Nail, PhD, RN, FAAN ${ }^{5}$
}

\begin{abstract}
Background: Millions of people with chronic illness suffer from fatigue. Fatigue is a complex, multidimensional symptom with poorly understood causes, wide variations in severity among individuals, and negative effects on multiple domains of daily life. Many patients with fatigue report the use of herbal remedies. Ginseng is one of the most widely used because it is believed to improve energy, physical and emotional health, and well-being.

Objective: To systematically review the published evidence to evaluate the safety and effectiveness of the two types of Panax ginseng (Asian [Panax ginseng] and American [Panax quinquefolius]) as treatments for fatigue.

Design: PubMed, CINAHL (Cumulative Index to Nursing and Allied Health), Ovid MEDLINE, and EMBASE databases were searched using Medical Subject Heading and keyword terms, including ginseng, Panax, ginsenosides, ginsenoside* (wild card), fatigue, fatigue syndrome, cancer-related fatigue, and chronic fatigue. Studies were included if participants had fatigue, had used one of the two Panax ginsengs as an intervention, and had scores from a self-report fatigue measure. Two reviewers independently assessed each article at each review phase and met to develop consensus on included studies. Risk of bias was assessed using version 5.3 of the Cochrane Collaboration Review Manager (RevMan), and results were synthesized in a narrative summary.

Results: The search strategy resulted in 149 articles, with 1 additional article located through review of references. After titles, abstracts, and full text were reviewed, 139 articles did not meet inclusion criteria. For the 10 studies reviewed, there was a low risk of adverse events associated with the use of ginseng and modest evidence for its efficacy.

Conclusions: Ginseng is a promising treatment for fatigue. Both American and Asian ginseng may be viable treatments for fatigue in people with chronic illness. Because of ginseng's widespread use, a critical need exists for continued research that is methodologically stronger and that includes more diverse samples before ginseng is adopted as a standard treatment option for fatigue.
\end{abstract}

Keywords: complementary and integrative health, herbal, Panax, symptom management

\section{Introduction}

$\mathbf{M}$ ILLIONS OF PEOPLE in the United States experience fatigue as a symptom of chronic illness or as an adverse event (AE) of the treatment of chronic illness, or both. ${ }^{1,2}$ Fatigue is a complex, multidimensional symptom with poorly understood causes, wide variations in severity among indi- viduals, and negative effects on multiple domains of daily life. ${ }^{3}$ Negative effects include decreased work productivity, physical activity, social interaction, and recreational activity, as well as feelings of loss and sadness. ${ }^{4}$

Over $32 \%$ of the population of the United States uses complementary and alternative medicine, ${ }^{5}$ with ginseng being on the top-10 list of the most-used natural products. ${ }^{6}$ People

\footnotetext{
${ }^{1}$ Department of Nursing, Mayo Clinic, Phoenix, AZ.

Divisions of ${ }^{2}$ Integrative Medicine and Health and ${ }^{3}$ Women's Health Internal Medicine, Mayo Clinic, Scottsdale, AZ.

${ }^{4}$ Library Services, Mayo Clinic, Scottsdale, AZ.

${ }^{5}$ School of Nursing, Oregon Health \& Science University, Portland, OR.

*Student at Oregon Health \& Science University, Portland, OR.
} 
who experience fatigue often report the use of herbal remedies, ${ }^{7}$ and one of the most commonly used is ginseng. Traditional Chinese Medicine and herbal medicine philosophies consider ginseng to be an adaptogen that helps restore balance to the body. Ginseng is believed to improve energy, physical and emotional health, and well-being. ${ }^{8-10}$ In the Panax genus (Asian [Panax ginseng] and American [Panax quinquefolius]), the root is the part of the plant with medicinal properties.

Asian and American ginseng both contain ginsenosides, which are the active compounds believed to act on the central nervous system ${ }^{11}$ and to have antioxidant ${ }^{12}$ and antiinflammatory properties, ${ }^{8}$ as well as cortisol-modulating effects. ${ }^{8}$ These are some of the reasons why researchers have considered $P$. ginseng to be a potential treatment for fatigue in multiple populations with chronic illness, including cancer survivors, patients with fibromyalgia, and patients with multiple sclerosis. The accepted and studied dosages of Panax ginseng range from 500 to $2000 \mathrm{mg}$. Knowing whether ginseng is a safe and effective treatment for fatigue in people with physical illness is important for clinicians and patients.

We located one published meta-analysis of the efficacy of ginseng supplements by using our search strategy and limiting it to reviews that assessed ginseng as a treatment for fatigue. ${ }^{13}$ The meta-analysis included 12 randomized controlled trials designed to determine whether ginseng reduced fatigue and improved physical performance. However, the analysis was limited by its broad scope. The studies reviewed included subjects who were not experiencing fatigue or who did not have physical illness, included outcomes based on self-report and physical performance, and combined all results into a single outcome for the analysis. Therefore, we aimed to expand on this work by evaluating the safety and effectiveness of the two types of Panax ginseng $(P$. ginseng and $P$. quinquefolius) as treatments for fatigue, focusing only on studies that included fatigued participants with a chronic illness and employed a self-report measure for fatigue, to assess the state of knowledge about the safety and effectiveness of Asian and American ginseng in managing fatigue in people with chronic illness. $^{14,15}$

\section{Methods}

The National Library of Medicine's (NLM) PubMed, CINAHL (Cumulative Index to Nursing and Allied Health), Ovid MEDLINE, and EMBASE databases were searched with no start date limit to April 2016 and limited to English language, clinical trials. The search was conducted using MeSH (Medical Subject Heading) and keyword terms, including ginseng, Panax, ginsenosides, ginsenoside* (wild card), fatigue, fatigue syndrome, cancer-related fatigue (CRF), and chronic fatigue (Box 1 includes the detailed search strategy). Studies were included if participants had fatigue, one of the two Panax ginsengs were used as an intervention, either as a single agent or in combination with other natural products, and scores from selfreport measures of fatigue outcomes were reported.

Studies that did not use a self-reported fatigue measure or that included only participants without fatigue (healthy subjects), or both, were excluded because the aim of this review was to assess the impact of ginseng as a treatment for those experiencing fatigue associated with chronic illness.

\begin{tabular}{|l|}
\hline Box 1. SEARCH STRATEGY \\
\hline For the literature search, the following databases were used: \\
the National Library of Medicine's PubMed, CINAHL \\
(Cumulative Index to Nursing and Allied Health), Ovid \\
MEDLINE, and EMBASE. All searches were limited to \\
English language and the following types of studies: \\
clinical trials, controlled clinical trials, randomized \\
controlled trials, meta-analyses, and systematic reviews. \\
The search results were as follows: PubMed, 22 citations; \\
CINAHL, 27 citations; Ovid MEDLINE, an additional \\
24 citations; and EMBASE, an additional 127 citations- \\
total, 200 citations. All results were put into an EndNote \\
file and 52 duplicate citations were removed, leaving 148 \\
citations. \\
Subject headings used: panax ginsenosides; fatigue; \\
fatigue syndrome, chronic; neoplasms. \\
Key/text words used: ginseng, ginsenosides, fatigue, \\
chronic fatigue, cancer-related fatigue, cancer.
\end{tabular}

Additionally, self-reported measures of fatigue aligned with the accepted definition that fatigue is a subjective, multidimensional symptom. ${ }^{16,17}$ Studies that reported results of non-Panax ginsengs (e.g., Siberian ginseng) were excluded because they have different compositions of active ingredients and do not contain ginsenosides. ${ }^{18}$

\section{Results}

The search strategy found 149 articles after 52 duplicates were removed, and 1 additional article was located through review of references (Fig. 1). Two assessors (N.M.A. and L.M.N.) independently reviewed each article at each review phase and met to develop consensus on included studies. After reviewing titles and abstracts, 134 articles did not meet inclusion criteria, resulting in 15 articles retrieved for review. Five of the articles retrieved were excluded for not including a Panax ginseng (2), not reporting fatigue as an outcome (1), or including only subjects without fatigue (2). Therefore, 10 articles were included in this review (Table 1). $8,11,12,19-25$

These studies are too heterogeneous to conduct a meaningful meta-analysis (doses, populations, and length of treatment vary widely). This review is not limited to randomized controlled trials or single-agent studies to assess the current state of evidence for ginseng as a treatment for fatigue. Risk of bias was assessed using version 5.3 of the Cochrane Collaboration Review Manager (RevMan), and results were synthesized in a narrative summary categorized by the type of ginseng tested: four American ginseng (P. quinquefolius), ${ }^{8,19-21}$ and six Asian ginseng $\left(P\right.$. ginseng). ${ }^{11,2,22-25}$

\section{American ginseng (P. quinquefolius)}

American ginseng is native to Canada and the United States. Four trials evaluated the impact of American ginseng on fatigue. ${ }^{8,19-21}$ One study evaluated the impact of American ginseng within a natural product combination (Immune No. 2) on symptoms, which included fatigue. ${ }^{21}$ Each study used a different fatigue measure, including the Brief Fatigue Inventory, Multidimensional Fatigue Symptom InventoryShort Form, Fatigue Severity Scale, and the Scores of Symptoms and Signs. ${ }^{8,19-21}$ 
FIG. 1. PRISMA flow diagram.

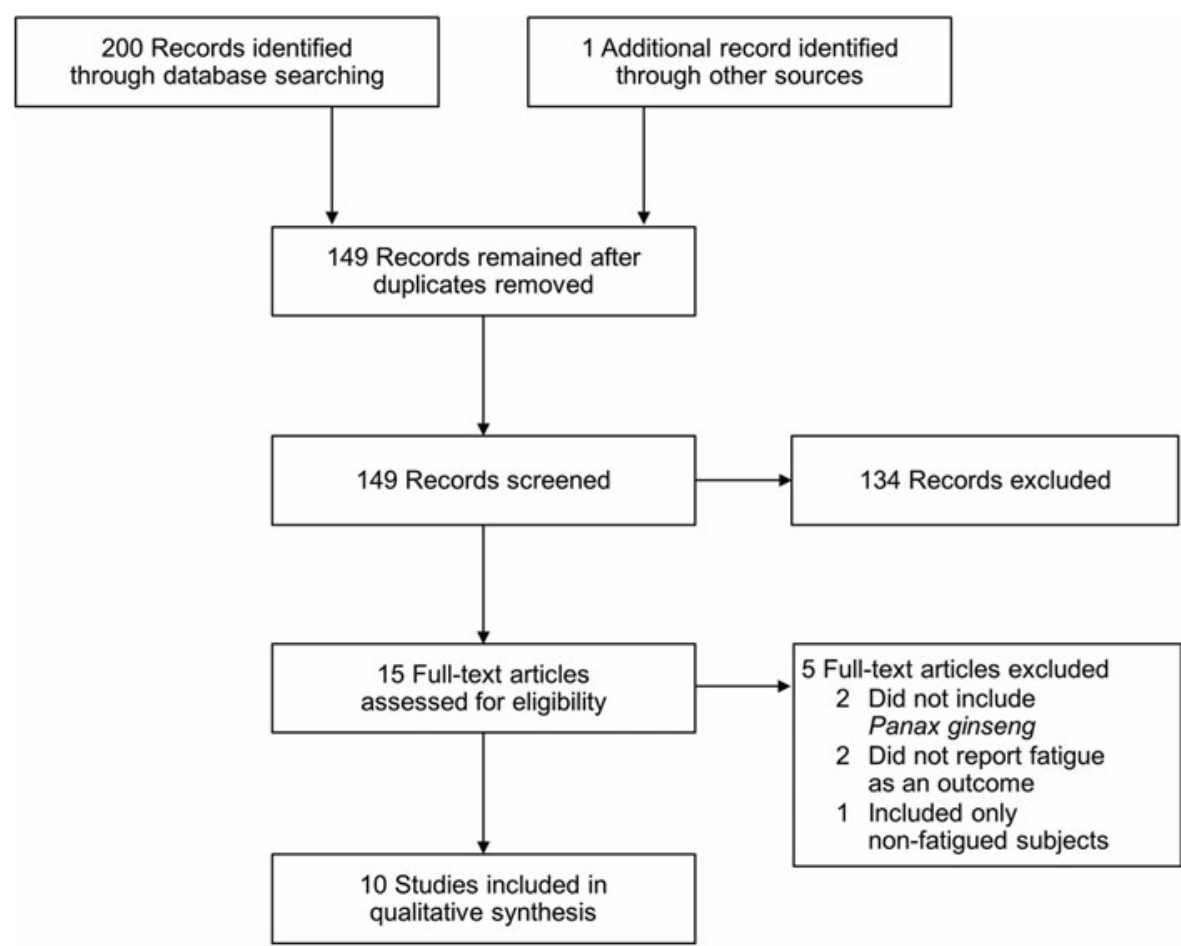

\section{Design}

All four American ginseng trials used bias controls, including double-blinding and a placebo. ${ }^{8,19-21}$ Three trials ranged from 8 weeks to 6 months. ${ }^{8,19,21}$ One study was a crossover trial that ran 6 weeks followed by a 2 -week washout, and then the crossover for an additional 6 weeks. ${ }^{20}$ Two of the studies had primary aims of evaluating the safety of ginseng and included multiple dosages of ginseng in the trials. ${ }^{19,20}$ Two of the studies included $2000 \mathrm{mg}$ per day as their highest dose of American ginseng, ${ }^{8,19}$ and one study had a maximum dose of $400 \mathrm{mg}$ per day of American ginseng. ${ }^{20}$ The study testing the Immune No. 2 combination did not include information about the ginseng dose used. ${ }^{21}$

\section{Sample}

Three of the four studies were conducted in the United States, ${ }^{8,19,20}$ and one was conducted in China. ${ }^{21}$ The sample consisted of cancer survivors in two studies, patients with multiple sclerosis in one study, and patients with HIV in the combination study. All participants were adults. ${ }^{8,19-21}$ Three studies included only those who reported fatigue for 1 month or more. ${ }^{8,19,20}$ Sample sizes ranged from 56 to 364 . One study with 282 participants was described as a pilot study; however, it was powered at $80 \%$, with an effect size of $0.41 .^{19}$ The smallest sample $(n=56)$ was from a study that employed a crossover design and was powered at $80 \%$, with an effect size of $0.8 .^{20}$ Three studies included mostly white women..$^{8,19,20}$ One study had mostly male participants $(62 \%) .{ }^{21}$ Attrition ranged from $12 \%$ to $39 \%$, with the largest attrition rate occurring in the cancer survivors' pilot study. ${ }^{19}$

\section{Safety and efficacy}

All dosages of American ginseng were tolerated well, with no serious AEs reported. Three of the four studies showed no differences in $\mathrm{AE}$ rates between the ginseng and placebo groups, ${ }^{8,19,21}$ two of which used $2000 \mathrm{mg}$ of ginseng as the highest dose. ${ }^{8,19}$ None reported serious AEs (i.e., lifethreatening events, death, or inpatient hospitalization). AEs reported included nausea, insomnia, headache, rash, and flulike symptoms. ${ }^{8,19,20}$ All four studies showed improvements in fatigue over time; however, only the 8-week 2000-mg trial and the 6-month American ginseng combination (Immune No. 2) demonstrated significant improvements in the primary fatigue outcome in the intervention group compared with that in the control group. ${ }^{8,21}$ However, the 400-mg trial did show a statistically significant improvement in the realtime digital fatigue scale for those who received ginseng compared with controls, but the study's authors did not believe this result was clinically meaningful. ${ }^{20}$

\section{Appraisal of the reviewed American ginseng research}

Overall, the study designs were strong because they controlled for multiple types of bias (Figs. 2 and 3). Three trials were powered at $80 \%$ or greater. $8,19,20$ Three of the four studies demonstrated significant improvements in fatigue in the intervention group compared with the control group. ${ }^{8,20,21}$ One study's authors did not believe their results to be clinically meaningful, but this was difficult to assess because they reported only a combined baseline (intervention and control groups together) fatigue score. ${ }^{20}$ The valid and reliable digital fatigue scale that tracked self-reported fatigue in real time by using a watch-like device ${ }^{20}$ has the potential to inform our understanding of fatigue patterns because it provides more data than the traditional self-report approach, which requires participants to think back over days, weeks, or months.

In one study, it was not clear if the fatigue evaluation was valid or reliable, which could undermine the results. ${ }^{21}$ One study completed a per-protocol analysis because two 


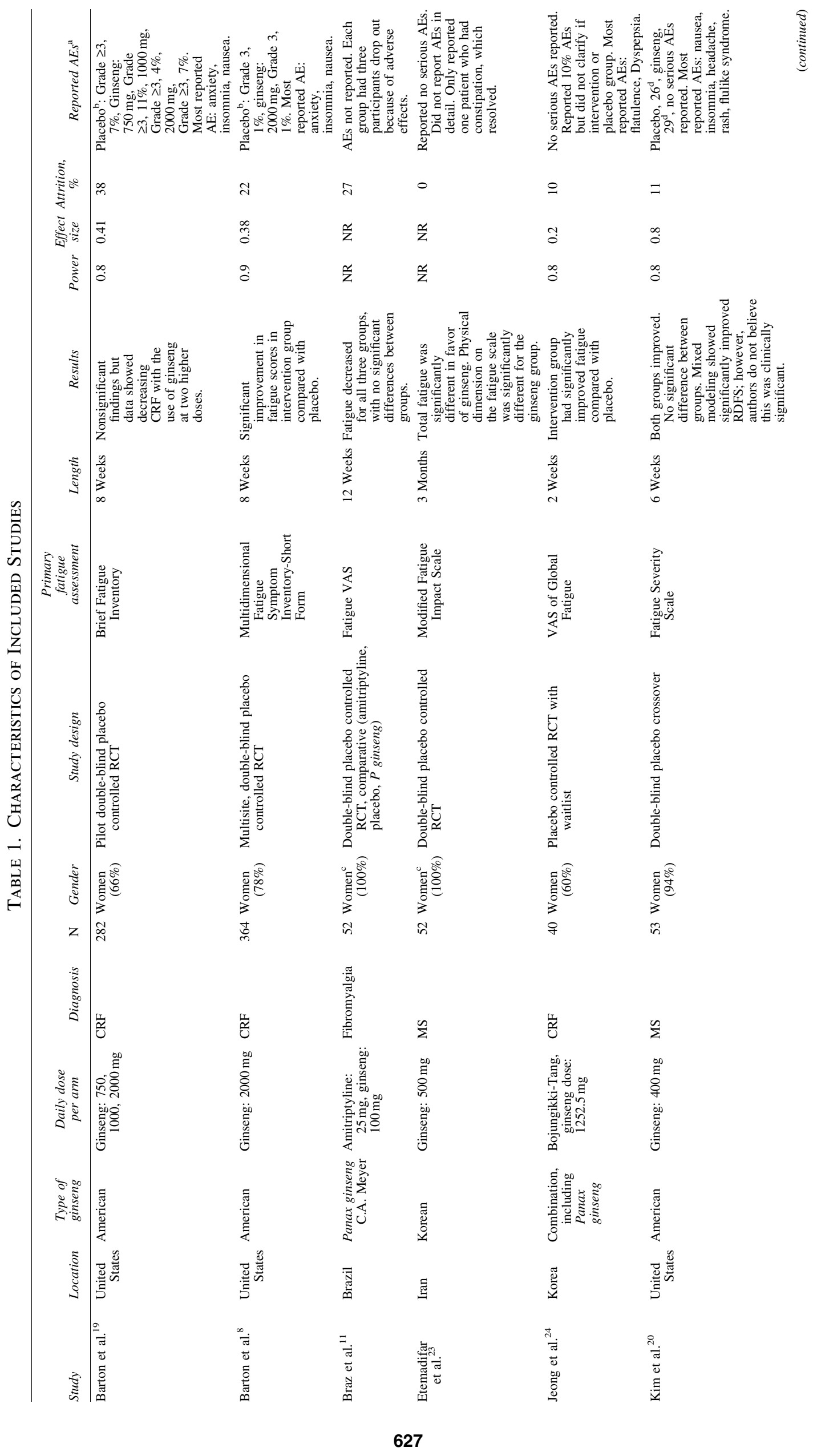




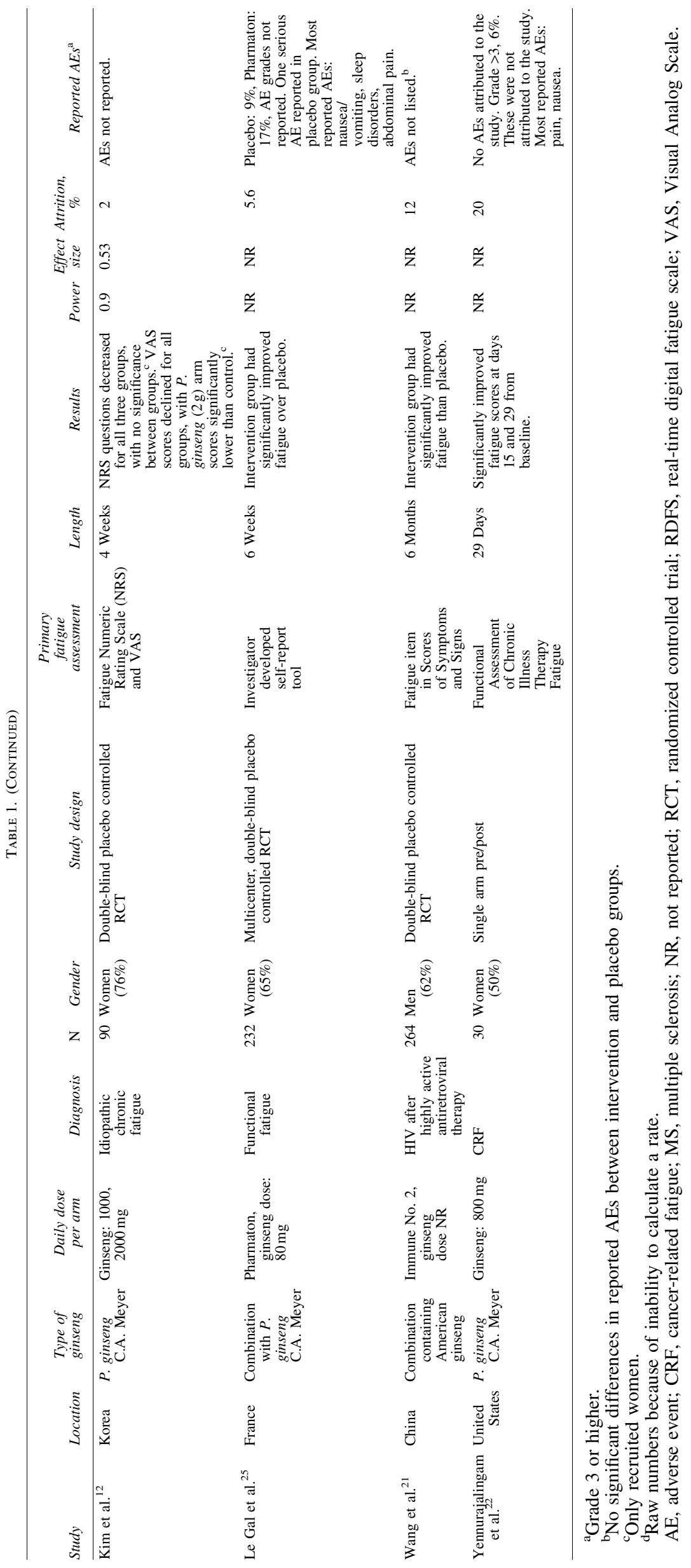




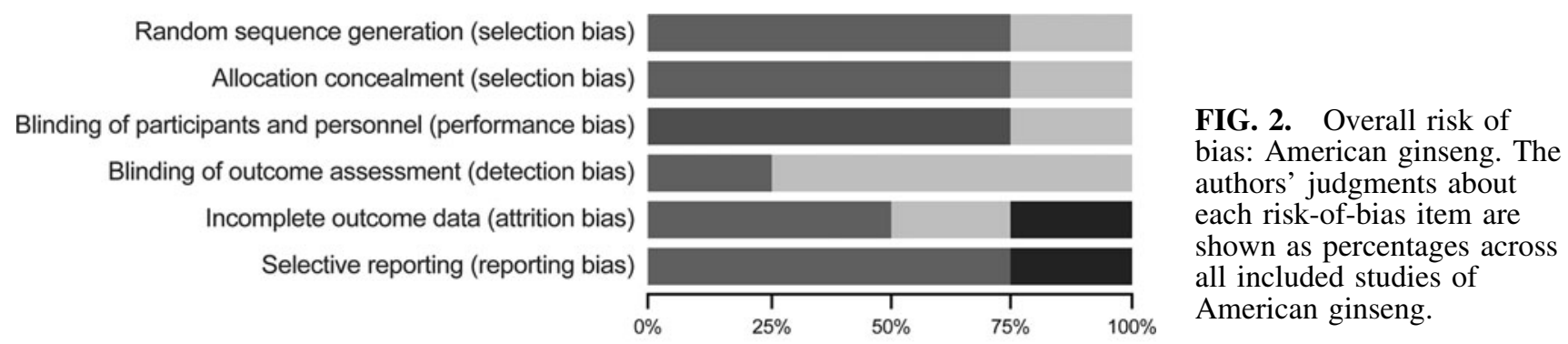

Low risk of bias

Unclear risk of bias

High risk of bias

participants were removed for not taking their ginseng, ${ }^{21}$ which could have led to a bias in missing data. Furthermore, one study found significant results testing a combined treatment (Immune No. 2) that included American ginseng. ${ }^{21}$ Testing a combination of ingredients produces results that cannot be attributed to any of the individual components. None of the study reports revealed any significant safety concerns.

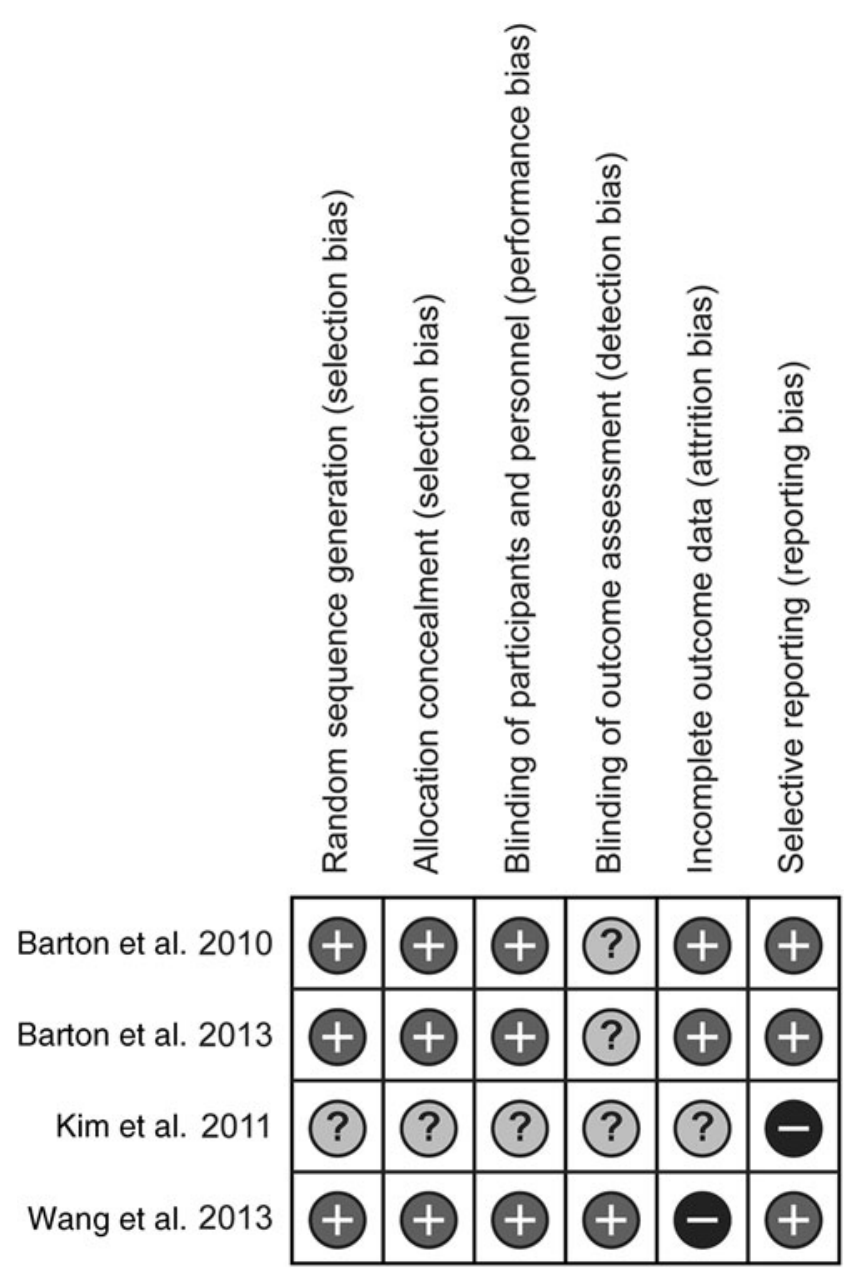

FIG. 3. Risk of bias: American ginseng. The authors' judgments about each risk-of-bias item are shown for each included study of American ginseng. +, Indicates low risk of bias; -, high risk of bias; ?, unclear risk of bias.

\section{Asian ginseng (P. ginseng)}

Asian ginseng is found in China, Korea, and Russia and is believed to be one of the most-researched species of ginseng. ${ }^{26}$ Six studies evaluated Asian ginseng as a treatment for fatigue. Four trials tested Asian ginseng $(P$. ginseng C.A. Meyer ${ }^{11,12,22}$ or $P$. ginseng [Korean ginseng]). ${ }^{23}$ Two studies tested Asian ginseng as part of a combination of natural products (Bojungikki-tang and Pharmaton). ${ }^{24,25}$ Multiple fatigue assessments were utilized, including a Visual Analog Scale, ${ }^{11,12,24}$ the Modified Fatigue Impact Scale, ${ }^{23}$ a Numeric Self-Rating Scale, ${ }^{12}$ the Functional Assessment of Chronic Illness Therapy-Fatigue, ${ }^{22}$ and one investigator-developed, self-report tool. ${ }^{25}$

\section{Design}

Four of the six studies were double-blind, placebo-controlled trials. ${ }^{11,12,23-25}$ One study was a randomized controlled trial with a waitlist control. ${ }^{24}$ One study was a single-arm, prospective, open-label trial. ${ }^{22}$ Overall, risk of bias was found to be unclear to high (Figs. 4 and 5). Study durations ranged from 4 weeks to 3 months. ${ }^{11,12,22-25}$ Daily doses ranged from 80 to $2000 \mathrm{mg}$ of $P$. ginseng, ${ }^{11,12,22-25}$ with the longest trial using a $250-\mathrm{mg}$ dose twice daily, for a total daily dose of $500 \mathrm{mg}$, for 3 months. ${ }^{23}$ Five of the six studies were designed to evaluate the efficacy of ginseng as a treatment for fatigue,,${ }^{11,12,23-25}$ and one was designed to evaluate the safety and tolerability of ginseng as a treatment for fatigue. ${ }^{22}$

\section{Sample}

The six studies were conducted in different countries. ${ }^{11,12,22-25}$ Study participants had the following diagnoses: cancer, ${ }^{22,24}$ fibromyalgia, ${ }^{11}$ chronic fatigue, ${ }^{12}$ multiple sclerosis, ${ }^{23}$ and functional fatigue. ${ }^{25}$ All studies included only adults. Four of six studies required participants to report fatigue as an inclusion criteria. ${ }^{12,22,24,25}$ Sample sizes ranged from 30 to 232 . The trial with the smallest sample $(n=30)$ was reported as a preliminary study. ${ }^{22}$ One study that was reported as a pilot study $(n=40)$ was powered at $80 \%$, with an effect size of $0.2 .^{24}$ None of the other studies reported the power of the study to detect change. Two of the six studies included only women. ${ }^{11,23}$ Three additional studies included mostly women: $60 \%, 76 \%$, and $65 \%$, respectively. ${ }^{12,24,25}$ The study conducted in the United States was the only one to report race; most participants were white $(84 \%) .{ }^{22}$ The lowest attrition rate was $0 \%,{ }^{23}$ with attrition rates in the other five studies reported as a minimum of $2 \%$ and a maximum of $27 \%$. $^{11,12,22,24,25}$ 
FIG. 4. Overall risk of bias: Asian ginseng. The authors' judgments about each risk-ofbias item are shown as percentages across all included studies of Asian ginseng.
Random sequence generation (selection bias)

Allocation concealment (selection bias)

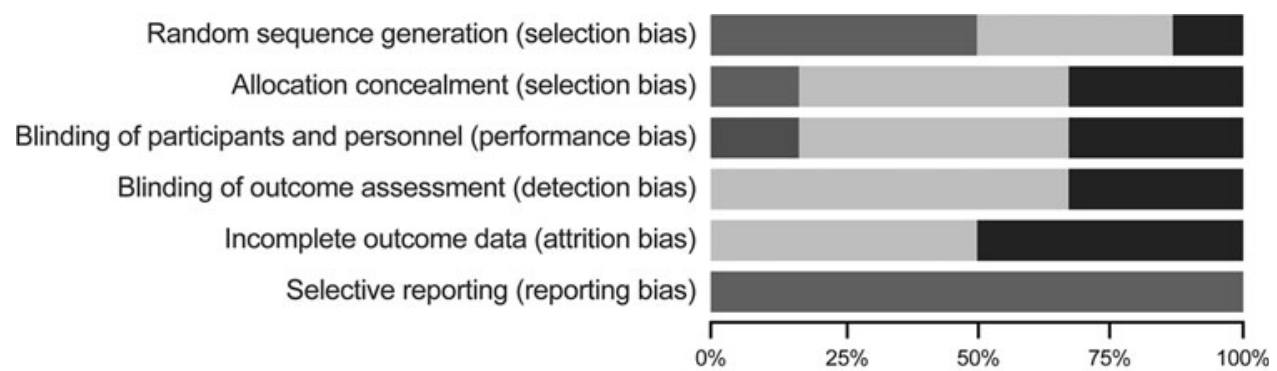

Low risk of bias $\square$ Unclear risk of bias $\square$ High risk of bias

\section{Safety and efficacy}

No study reported serious AEs in the intervention group. ${ }^{11,12,22-25}$ One study that tested ginseng in a natural product combination (Pharmaton) reported a single, serious AE in the control group; a participant had to be admitted to the hospital for edema of the uvula that resolved after corticosteroid therapy. ${ }^{25}$ The single-arm study reported a $6 \%$ rate for serious AEs; however, none was attributed to ginseng. ${ }^{22}$ One study did not report any AEs, but the investigators noted that nine participants (three from each arm) dropped out be-

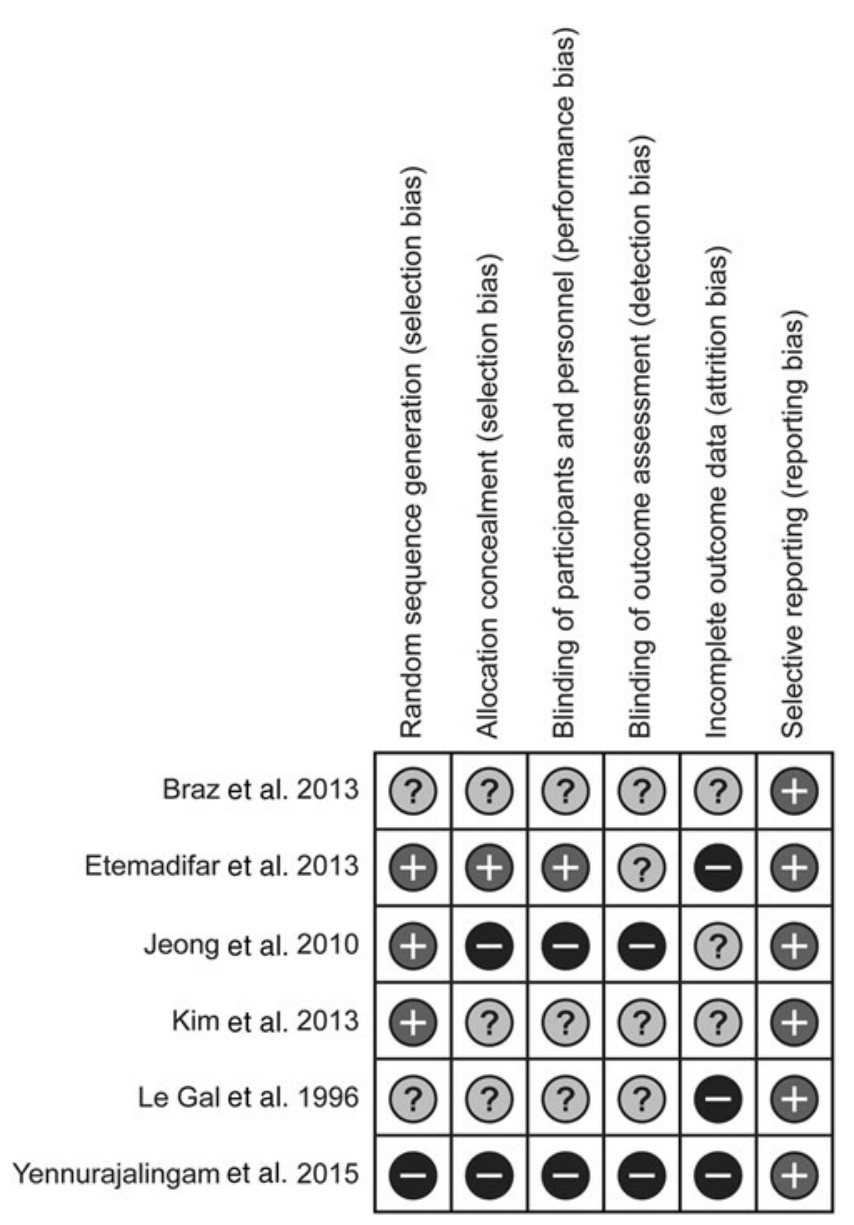

FIG. 5. Risk of bias: Asian ginseng. The authors' judgments about each risk-of-bias item for each included study of Asian ginseng. +, Indicates low risk of bias; -, high risk of bias; ?, unclear risk of bias. cause of AEs. ${ }^{11}$ Only three of the six studies reported AEs in detail, with rates that ranged from $10 \%$ to $53 \% .^{22,24,25}$ In the study that reported the largest $\mathrm{AE}$ rate $(53 \%)$, none of the AEs was attributed to the intervention. ${ }^{22}$ The most-reported AEs included nausea/vomiting, sleep disorders, abdominal pain, and bowel disorders. ${ }^{12,22-25}$ Fatigue decreased in all six studies, with five studies reporting significant differences in fatigue measures in the invention group..$^{12,22-25}$

\section{Appraisal of reviewed Asian ginseng research}

Most (four of six) of the studies were double-blind, placebocontrolled trials; however, they did not report how they mitigated bias (Figs. 4 and 5). ${ }^{11,12,23,25}$ Four of the six studies showed that the intervention group had significantly improved fatigue outcomes compared with the placebo group ${ }^{12,22-25}$; the single-arm prospective, open-label trial showed significantly improved fatigue scores at 15 and 29 days compared with fatigue at baseline. ${ }^{22}$ The single-arm trial also had the highest risk of bias. ${ }^{22}$ Because this study did not have a control group, it is possible that the improvement over time resulted from something other than the ginseng treatment. ${ }^{22}$

Three studies excluded participants from the analysis if they did not adhere to the study protocol, thus a per-protocol analysis was done rather than an intent-to-treat analysis. ${ }^{22,23,25}$ A per-protocol analysis may result in bias related to missing data. ${ }^{27}$ However, one study reported no attrition, but it is not clear if the attrition count was defined as including participants who were excluded for not following the study protocol. ${ }^{23}$ One study used an investigator-developed fatigue measure, but did not report validity or reliability of the measure. ${ }^{25}$ If the fatigue measure was not valid and reliable, this would challenge the validity of the results. ${ }^{25}$ Furthermore, two studies tested Asian ginseng in a combination with other natural products, making it difficult to ascertain the relative contribution of the ingredients to the study outcomes. $^{24,25}$ None of the studies reported significant safety concerns for the intervention group.

\section{Cancer-related fatigue}

A subanalysis was conducted for the studies of CRF because they made up the largest illness group in this review. Four of the 10 studies evaluated ginseng's impact on CRF. ${ }^{8,19,22,24}$ Two studies included both active treatment and post-treatment cancer survivors. ${ }^{8,19}$ One study included only results at 2 months after treatment, ${ }^{24}$ and the other included only active-treatment cancer survivors. ${ }^{22}$ 


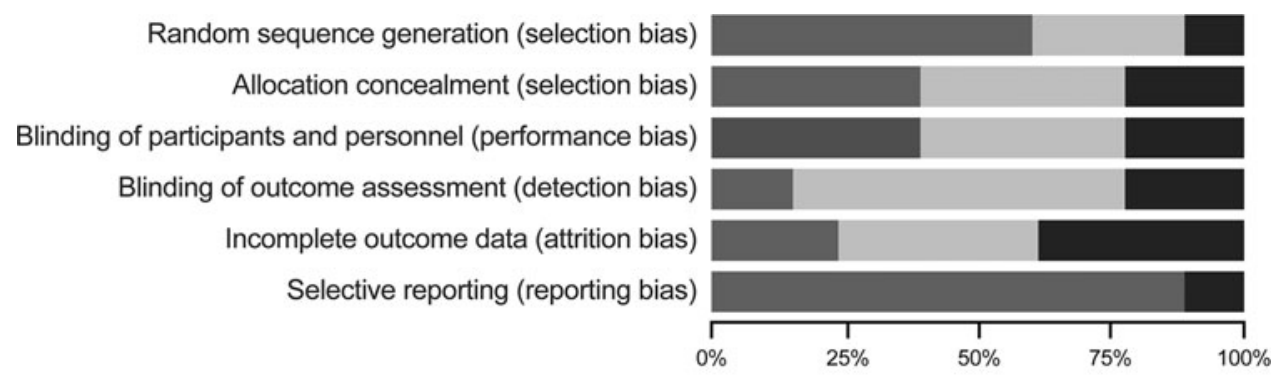

Low risk of bias
Unclear risk of bias
High risk of bias
FIG. 6. Risk of bias: the authors' judgments about each risk-of-bias by percentage across all included studies.
Ginseng doses ranged from 800 to $2000 \mathrm{mg}$. Ginseng doses in three of the four studies were $>1000 \mathrm{mg}$, and intervention periods ranged from 2 to 8 weeks. ${ }^{8,19,24}$ Three of the four CRF studies reported significant results. ${ }^{8,22,24}$ Two showed that ginseng (one, $P$. quinquefolius; one, $P$. ginseng) improved fatigue significantly in the study groups versus the controls, ${ }^{8,24}$ whereas the other study showed that $P$. ginseng significantly improved participants' fatigue scores from those at baseline. 22

Although the studies were promising for their effectiveness in decreasing CRF, the results should be approached cautiously. Two of the four studies had sample sizes of fewer than 50 participants. $^{22,24}$ Three had mostly women participants. ${ }^{8,19,24}$ Both the sample size and sample composition may have impacted the generalizability of the results. To date, none of the positive results of the CRF ginseng studies has been replicated.

\section{Discussion}

This review aimed to evaluate the safety and effectiveness of the two types of Panax ginseng (Asian $[P$. ginseng] and American [P. quinquefolius]) as a treatment for fatigue through examining study designs, samples, safety, and efficacy. Key strengths of the studies included the study designs and the safety and efficacy results. Most of the studies (8/10) were double-blind, randomized controlled trials,, ,11,12,19-21,23,25 which allows for control of multiple types of bias; however, mitigating detection and attrition bias would increase the overall strength of the evidence (Figs. 6 and 7). Four (4/10) studies reported being powered at $80 \%$ or greater, which can add to the strength of the studies to detect the effects of the treatment. ${ }^{8,19,20,24}$

None of the studies reported significant AEs attributed to ginseng. One study did report a serious $\mathrm{AE}$ attributed to the placebo. ${ }^{25}$ Reported rates of grade $\geq 3$ AEs ranged from $1 \%$ to $11 \%$. ${ }^{8,19,22,24,25}$ Three of 10 studies showed no significant differences in AEs between the ginseng and placebo groups. . $^{8,19,21}$ The most common, nonserious AEs reported were nausea/ vomiting, sleep disorders, abdominal pain, and bowel disorders.

Most (7/10) studies showed significant improvements in fatigue scores in the intervention group compared with the control group. $8,12,20,21,23-25$ Additionally, one single-arm study showed significant improvement in fatigue scores from baseline at 15 and 29 days $^{22}$; however, this study did not have a control group. Therefore, there could be plausible explanations other than treatment with ginseng for the improvement in fatigue over time. ${ }^{22}$ Furthermore, one study's authors interpreted their findings as not being clinically meaningful; however, because the authors reported a combined (placebo and intervention) baseline for fatigue, it was difficult to assess the rationale for their conclusion. ${ }^{20}$

Five of the six studies reporting significant differences in fatigue between the ginseng and placebo groups may have

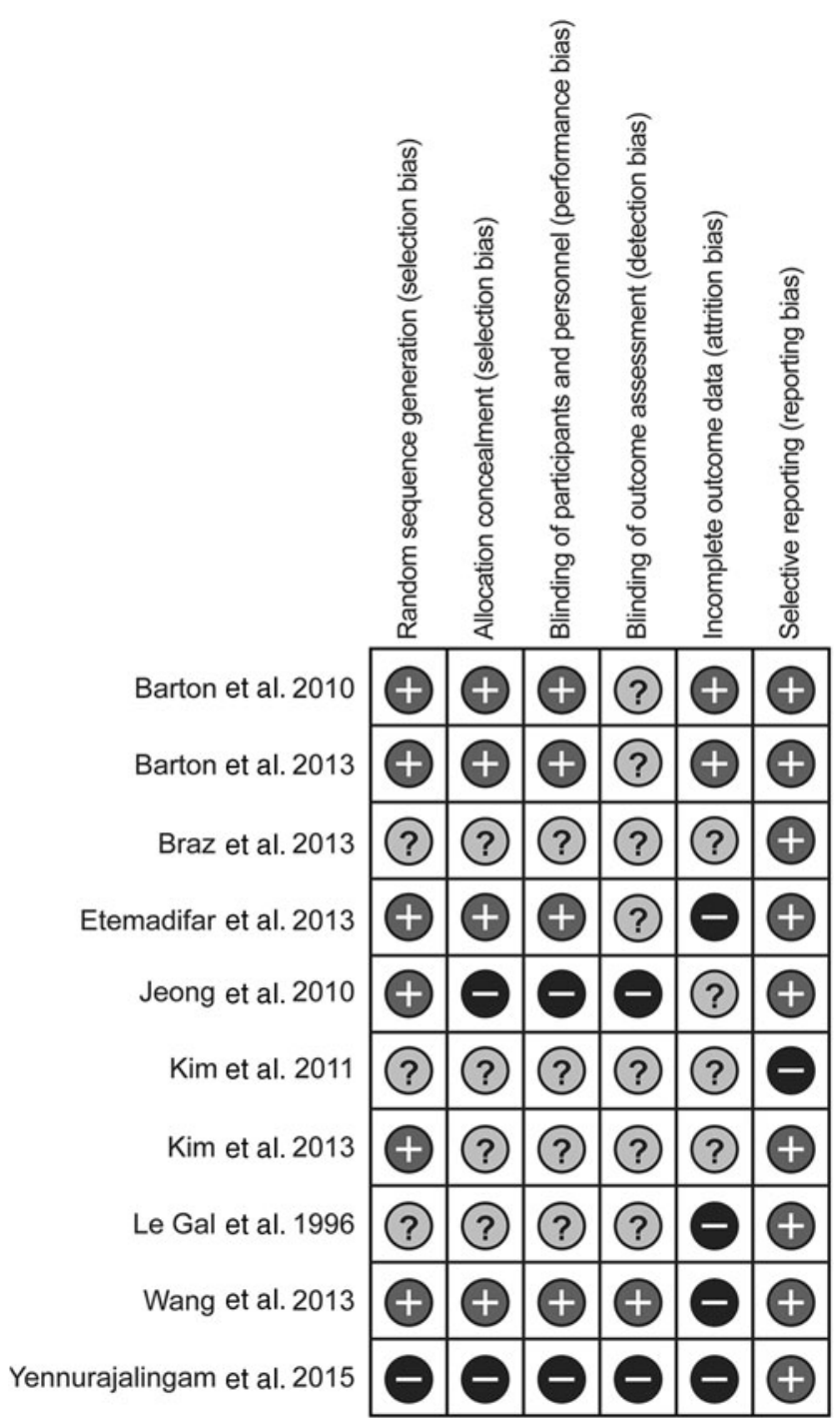

FIG. 7. Risk of bias. The authors' judgments about each risk-of-bias item for each included study. + , Indicates low risk of bias; -, high risk of bias; ?, unclear risk of bias. 
been biased from missing data, because they completed perprotocol analyses and/or did not seem to adhere to intentionto-treat analysis. ${ }^{12,21-23,25}$ This type of analysis limits the ability to overcome biases related to attrition or missing data, or both, which could favor the intervention group. ${ }^{27}$ Two studies did not show significant improvements in fatigue: one was a dose-finding pilot study, ${ }^{19}$ and the other was a threearm randomized controlled trial with 52 participants that tested the second lowest dose of ginseng $(100 \mathrm{mg}){ }^{11}$

Most of the studies (6/10) were conducted in Western countries. ${ }^{8,11,19,20,22,25}$ Sample sizes ranged from 30 to 364. ${ }^{8,11,12,19-25}$ Three (3/10) studies were either pilot or preliminary studies. ${ }^{19,22,24}$ Cancer was the most common (4/ 10) condition studied. ${ }^{8,19,22,24}$ Eight $(8 / 10)$ of the studies included mostly women participants. . $^{8,11,12,19,20,23-25}$ Two studies' inclusion criteria specified that participants had to be women. ${ }^{11,23}$ Because of the preponderance of women participants, the study results cannot necessarily be generalized to men-possibly because women may be more likely to seek alternative treatments, such as ginseng, or because the conditions studied primarily impacted women (e.g., fibromyalgia and multiple sclerosis). ${ }^{28,29}$

No consistent dosage for ginseng was used. Study interventions varied from 80 to $2000 \mathrm{mg}$ daily. ${ }^{8,11,12,19-25}$ Three of the 10 studies used $2000 \mathrm{mg}$ as the maximum dose. ${ }^{8,12,19}$ All but two (six of eight) studies that demonstrated significant improvements in fatigue in the intervention group tested gin-

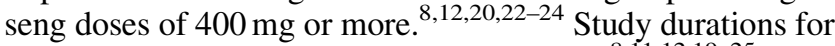
treatment ranged from 2 weeks to 6 months. ${ }^{8,11,12,19-25}$ Most (seven of eight) studies that showed significant improvements

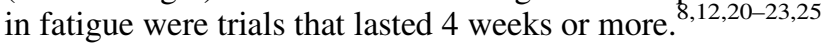

\section{Limitations}

One limitation of this review is limiting the studies to English language only, which could have led to a language bias. However, in a meta-analysis, Morrison et al. ${ }^{30}$ showed that although limiting to English only in reviews is perceived to lead to a language bias, a language limitation in search strategy did not result in systematic bias. Additionally, not limiting this review to single-agent use of ginseng may have confounded the results regarding efficacy of ginseng as a treatment for fatigue. However, limiting the studies to singleagent use of ginseng would have ignored the traditional uses of ginseng (often multiagent combinations) and would have narrowed the assessment of the state of evidence on the effectiveness of ginseng for fatigue in people with chronic illness.

\section{Conclusion and clinical implications}

Overall, there was modest support for ginseng as a treatment for fatigue. Both American and Asian ginseng may be viable treatments for fatigue in people with chronic illness because of the low risk associated with its use, coupled with modest evidence for its efficacy. However, it is critical that future research build on the evidence provided by the studies reviewed about ginseng dose and duration of treatment, be methodologically strong, and include more diverse samples before ginseng, which is already in widespread use, is adopted as a standard treatment option for fatigue.

\section{Future research recommendations}

Future research should focus on developing safe and effective interventions for fatigue. We found three obvious gaps in the literature regarding American and Asian ginseng as treatments for fatigue in people with chronic illness that should be considered for additional research. First, future research reports should include a clear presentation of the statistical methods used and specify how missing data were handled to address attrition bias. Second, larger and more diverse samples are needed to increase the generalizability and statistical power of the studies. Third, future studies should be designed for a regimen of $400 \mathrm{mg}$ or more of ginseng for at least 4 weeks. Finally, the results of current studies with significant findings should be replicated by future studies to assure that the findings can be reproduced.

\section{Acknowledgments}

Funding for this article was received from the Jonas Center for Nursing Excellence, Mayo Clinic Nursing Research Steering Committee, and the Oregon Health \& Science University Presidential Scholarship.

\section{Author Disclosure Statement}

No competing financial interests exist.

\section{References}

1. Jiang SL, Liu HJ, Liu ZC, et al. Adjuvant effects of fermented red ginseng extract on advanced non-small cell lung cancer patients treated with chemotherapy. Chin J Integr Med 2017;23:331-337.

2. Centers for Disease Control and Prevention. Myalgic encephalomyelitis/chronic fatigue syndrome. 2013. Online document at: www.cdc.gov/me-cfs, accessed September 21, 2017.

3. Bower JE. Cancer-related fatigue-Mechanisms, risk factors, and treatments. Nat Rev Clin Oncol 2014;11:597-609.

4. Centers for Disease Control and Prevention. Basic information about cancer survivorship. 2015. Online document at: https://www.cdc.gov/cancer/survivorship/basic_info/sur vivors/index.htm accessed March 29, 2018.

5. National Center for Complementary and Integrative Health (NCCIH). Use of complementary health approaches in the U.S. 2017. Online document at: https://nccih.nih.gov/research/ statistics/NHIS/2012/key-findings, accessed March 29, 2018.

6. National Center for Complementary and Integrative Health (NCCIH). Use of complementary health approaches in the U.S. National Health Interview Survey (NHIS). 2016. Online document at: https:/nccih.nih.gov/research/statistics/ NHIS/2012, accessed March 29, 2018.

7. Cutshall SM, Cha SS, Ness SM, et al. Symptom burden and integrative medicine in cancer survivorship. Support Care Cancer 2015;23:2989-2994.

8. Barton DL, Liu H, Dakhil SR, et al. Wisconsin ginseng (Panax quinquefolius) to improve cancer-related fatigue: A randomized, double-blind trial, N07C2. J Natl Cancer Inst 2013;105:1230-1238.

9. Panossian A, Wikman G, Kaur P, Asea A. Adaptogens exert a stress-protective effect by modulation of expression of molecular chaperones. Phytomedicine 2009;16:617-622.

10. Coleman CI, Hebert JH, Reddy P. The effects of Panax ginseng on quality of life. J Clin Pharm Ther 2003;28:5-15. 
11. Braz AS, Morais LC, Paula AP, et al. Effects of Panax ginseng extract in patients with fibromyalgia: A 12-week, randomized, double-blind, placebo-controlled trial. Rev Bras Psiquiatr 2013;35:21-28.

12. Kim HG, Cho JH, Yoo SR, et al. Antifatigue effects of Panax ginseng C.A. Meyer: A randomised, double-blind, placebo-controlled trial. PLoS One 2013;8:e61271.

13. Bach HV, Kim J, Myung SK, Cho YA. Efficacy of ginseng supplements on fatigue and physical performance: A metaanalysis. J Korean Med Sci 2016;31:1879-1886.

14. Oncology Nursing Society. Fatigue. Oncology Nursing Society. 2017. Online document at: www.ons.org/practiceresources/pep/fatigue, accessed September 21, 2017.

15. National Comprehensive Cancer Network. Cancer-related fatigue. 2017. Online document at: www.nccn.org/professionals/ physician_gls/PDF/fatigue.pdf, accessed September 21, 2017.

16. Hann DM, Jacobsen PB, Azzarello LM, et al. Measurement of fatigue in cancer patients: Development and validation of the Fatigue Symptom Inventory. Qual Life Res 1998;7: 301-310.

17. Mota DD, Pimenta CA. Self-report instruments for fatigue assessment: A systematic review. Res Theory Nurs Pract 2006;20:49-78.

18. U.S. Fish and Wildlife. U.S. Fish and Wildlife, ITIS report: Panax L. 2017. Online document at: https://www.itis.gov/ servlet/SingleRpt/SingleRpt?search_topic=TSN\&search_value =29398\#null, accessed September 21, 2017.

19. Barton DL, Soori GS, Bauer BA, et al. Pilot study of Panax quinquefolius (American ginseng) to improve cancer-related fatigue: A randomized, double-blind, dose-finding evaluation: NCCTG trial N03CA. Support Care Cancer 2010;18: 179-187.

20. Kim E, Cameron M, Lovera J, et al. American ginseng does not improve fatigue in multiple sclerosis: A single center randomized double-blind placebo-controlled crossover pilot study. Mult Scler 2011;17:1523-1526.

21. Wang J, Li Y, Tang YL, et al. Effect of Immune No. 2 on the immune reconstitution in patients with HIV/AIDS after highly active antiretroviral treatment: A randomized double blind placebo controlled clinical trial. Chin J Integr Med 2013;19:340-346.
22. Yennurajalingam S, Reddy A, Tannir NM, et al. Highdose Asian ginseng (Panax ginseng) for cancer-related fatigue: A preliminary report. Integr Cancer Ther 2015; 14:419-427.

23. Etemadifar M, Sayahi F, Abtahi SH, et al. Ginseng in the treatment of fatigue in multiple sclerosis: A randomized, placebo-controlled, double-blind pilot study. Int J Neurosci 2013;123:480-486.

24. Jeong JS, Ryu BH, Kim JS, et al. Bojungikki-tang for cancer-related fatigue: A pilot randomized clinical trial. Integr Cancer Ther 2010;9:331-338.

25. Le Gal M, Cathebras P, Strüby K. Pharmaton capsules in the treatment of functional fatigue: A double-blind study versus placebo evaluated by a new methodology. Phytother Res 1996;10:49-53.

26. Kiefer D, Pantuso T. Panax ginseng. Am Fam Physician 2003;68:1539-1542.

27. Higgins JPT, Green S (editors). Cochrane Handbook for Systematic Reviews of Interventions. Version 5.1.0 [updated March 2011]. The Cochrane Collaboration, 2011. Online document at: http://handbook.cochrane.org.

28. Krasselt M, Baerwald C. Sex, symptom severity, and quality of life in rheumatology. Clin Rev Allergy Immunol. 2017. [Epub ahead of print]; DOI: 10.1007/s12016-017-8631-6.

29. Noseworthy JH, Lucchinetti C, Rodriguez M, Weinshenker BG. Multiple sclerosis. N Engl J Med 2000;343:938-952.

30. Morrison A, Polisena J, Husereau D, et al. The effect of English-language restriction on systematic review-based meta-analyses: A systematic review of empirical studies. Int J Technol Assess Health Care 2012;28:138-144.

Address correspondence to: Noël M. Arring, DNP, RN, OCN Department of Nursing Mayo Clinic 5777 E Mayo Blvd Phoenix, AZ 85054

E-mail: arring.noel@mayo.edu 